\title{
Helical magnetic ordering in thin FeGe membranes
}

\author{
D. M. Burn $\odot,{ }^{1, *}$ S. L. Zhang,,${ }^{2,3, \dagger}$ S. Wang,${ }^{4}$ H. F. Du,${ }^{4, \ddagger}$ G. van der Laan,${ }^{1,8}$ and T. Hesjedal $\odot^{3, \|}$ \\ ${ }^{1}$ Magnetic Spectroscopy Group, Diamond Light Source, Didcot OX11 ODE, United Kingdom \\ ${ }^{2}$ School of Physical Science and Technology, ShanghaiTech University, Shanghai 201210, China \\ ${ }^{3}$ Department of Physics, Clarendon Laboratory, University of Oxford, Oxford OX1 3PU, United Kingdom \\ ${ }^{4}$ Anhui Province Key Laboratory of Condensed Matter Physics at Extreme Conditions, High Magnetic Field Laboratory, \\ Chinese Academy of Sciences and University of Science and Technology of China, Hefei 230026, China
}

(Received 17 September 2019; published 1 November 2019)

\begin{abstract}
A detailed magnetic phase diagram of FeGe membranes with fields applied out-of-plane is determined using small-angle soft $\mathrm{x}$-ray elastic scattering. In addition to the well-established skyrmion and helical phase, we identify an additional ordered phase which partly coexists with the helical phase in a pocket beneath the skyrmion phase. Furthermore, an evolution of the modulation wave vector, $\mathbf{q}$, is observed when traversing the magnetic phase pocket. For the ordinary and the additional helical phase, $\mathbf{q}$ only varies with normalized field but not with temperature-in sharp contrast to the skyrmion phase, for which $\mathbf{q}$ is both field and temperature dependent. This study of the helical phases in thin FeGe membranes lays the foundation for their use in future magnetic storage and logic devices.
\end{abstract}

DOI: 10.1103/PhysRevB.100.184403

\section{INTRODUCTION}

Complex spin structures are one of the most interesting topics in magnetism, as they contain rich underlying physics, on both microscopic and macroscopic length scales, and as they can serve as efficient storage media for memory applications. FeGe is a noncentrosymmetric helimagnet (cubic space group $P 2{ }_{1} 3$ ) in which noncollinear spin textures result from a competition between the exchange interaction and the spin-orbit-induced Dzyaloshinskii-Moriya (DM) interaction. Among the known cubic chiral magnets, FeGe has a high ordering temperature of $T_{\mathrm{C}}=278 \mathrm{~K}$ [1-3] (only second to Mn-type Co-Zn-Mn alloy [4]-in comparison to $29 \mathrm{~K}$ for $\mathrm{MnSi}[5,6]$ and $170 \mathrm{~K}$ for MnGe [7,8]. Bulk FeGe has a well-established magnetic phase diagram showing ordered helical, conical, and skyrmion states [9]. The skyrmion pocket has a wide temperature vs field window reaching up to near room temperature $[10,11]$, which has been observed through multiple techniques, including Lorentz transmission electron microscopy (LTEM) [10,12]. Further, the dimensions of a FeGe sample also has strong influence on the phase diagram. For instance, when the thickness of the sample is reduced to be on the order of the skyrmion lattice parameter, the skyrmion lattice phase pocket increases significantly in terms of temperature and field $[10,13]$.

Topological aspects of complex spin structures were becoming the focus of interest in particular after the discovery

\footnotetext{
*david.burn@diamond.ac.uk

†zhangshl1@shanghaitech.edu.cn

¥duhf@hmfl.ac.cn

§Gerrit.vanderlaan@diamond.ac.uk

"thorsten.hesjedal@physics.ox.ac.uk
}

of two-dimensional solitonic skyrmion structures. However, spins can also form much simpler, yet still as topologically entangled structures, the so-called chiral soliton lattice (CSL) $[14,15]$. Such a structure can be regarded as the onedimensional version of the soliton solution, due to the competition among isotropic exchange, DM, and Zeeman interactions, whereby skyrmions represent the two-dimensional case. A CSL can be expected when a field is applied perpendicular to the modulation vector $\mathbf{q}$ in the helical phase. The helical spin structure contains alternating regions of spins aligned parallel and antiparallel to the applied field. Zeeman-energy minimization gives preference towards the spins aligned with the field and leads to an expansion of this region in the structure with respect to the antiparallel aligned spins. This results in a CSL structure with an associated reduction in $\mathbf{q}$ with an out-of-plane applied field $[14,15]$. The zero-field helical modulation period of $\lambda \approx 70 \mathrm{~nm}$ [1] is determined by the ratio $J / D$ of the exchange interaction $J$ and DM interaction $D$ (and nearly identical to the skyrmion lattice constant [7]). So far, helical ordering in FeGe thin films has only been studied with small-angle neutron scattering [16], and investigation of the field and temperature dependence of the ordered helical structures high q resolution has been lacking.

Here, we study the helical phase in FeGe in great detail using small-angle resonant elastic x-ray scattering (SAREXS). This technique with its inherent high resolution is particularly suited to detect the complex variations in $\mathbf{q}$ as the field vs temperature $(B-T)$ phase diagram for $\mathrm{FeGe}$ is traversed [17-20]. We find that the ratio of $J / D$ alone is insufficient to describe the magnetic winding within the helical (as well as the skyrmion) phase. Furthermore, we report the existence of an additional helical phase that is found to coexist between the skyrmion and helical pockets along a specific crystalline orientation. 

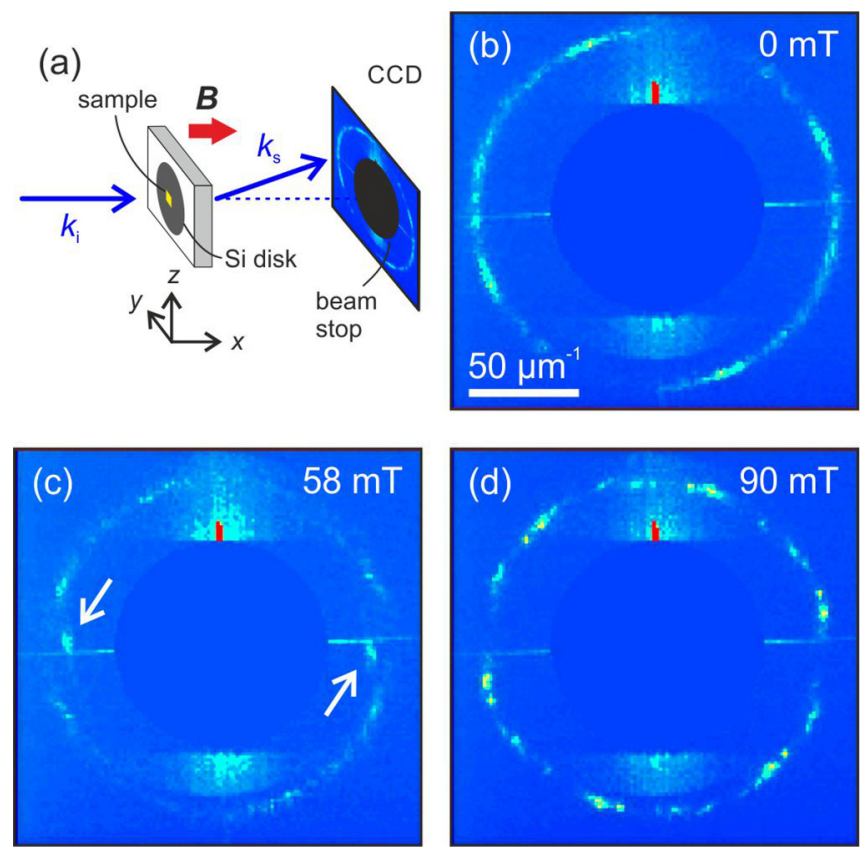

FIG. 1. Small angle x-ray scattering from a FeGe membrane with the magnetic field applied out-of-plane of the sample (along the $\mathrm{x}$-ray beam direction). (a) Experimental setup showing the scattering configuration (not to scale). (b-d) Representative scattering patterns observed at 0,58 , and $90 \mathrm{mT}$, respectively, at $257 \mathrm{~K}$. Patterns are dominated to first order by a bright ring, indicative of the disordered helical phase. Further, four-fold symmetric brighter arcs are due to locking of the helices in the sample plane because of magnetocrystalline anisotropy. In (c), two additional peaks are observed (indicated by white arrows) identified in this paper as a second helical phase, Hel-II. (d) At high fields, the system transforms into a multidomain skyrmion state.

\section{METHODS}

A 200-nm-thick FeGe membrane, measuring $20 \times$ $20 \mu \mathrm{m}^{2}$ in area, was milled from a single crystal using focused-ion-beam processing (see Ref. [21] for details). The membrane was transferred onto a 3-mm-diameter Si disk with a $10 \mu \mathrm{m} \times 10 \mu \mathrm{m}$ square aperture in the center, and secured in place with locally deposited Pt. The sample was studied using high-resolution SAREXS in transmission geometry, as depicted in Fig. 1(a), on beamline I10 at the Diamond Light Source (Oxfordshire, UK). Circularly polarized x-rays with energy tuned to the Fe $L_{3}$ absorption edge were scattered from the magnetic structure in the sample. This small-angle scattering was captured by a CCD detector with a sample to detector distance of $474 \mathrm{~mm}$, giving an angular resolution of $0.0016^{\circ}$ and $\mathbf{q}$ resolution of $1.6 \times 10^{-2} \mu \mathrm{m}^{-1}$ at the Fe $L_{3}$ edge. The sample was mounted on a He cryostat within an octupolar vector magnet, and the small-angle magnetic scattering was investigated as a function of both the temperature and the out-of-plane applied magnetic field.

\section{EXPERIMENTAL RESULTS}

Figures 1(b)-1(d) show typical small-angle scattering data collected at $257 \mathrm{~K}$ in different out-of-plane magnetic fields (field along the x-ray propagation direction). At zero field [Fig. 1(b)], the ring of scattered intensity with constant $|\mathbf{q}|$ originates from the helical phase. Here, the proper-screw spin structure has a fixed periodicity, and hence constant magnitude. However, as the crystal is cut in an arbitrary direction, the propagation directions of $\mathbf{q}$ are to first-order not fixed giving rise to a ring-shaped intensity distribution. Within the ring, some regions appear brighter, however, these features are not correlated with the cubic magnetocrystalline anisotropy, which is suppressed in thinned-down samples [22].

At an applied field of $58 \mathrm{mT}$ [Fig. 1(c)], a reduction in $\mathbf{q}$ accompanied by a decrease in scattered intensity is observed. Additionally, two peaks (indicated by the white arrows) appear along the near-horizontal axis at $\mathbf{q}$ values lower than those of the helical ring. These indicate the presence of a spin structure with a single propagation vector $\mathbf{q}$, which is aligned perpendicular to the film plane, similar to the one observed in epitaxial FeGe thin films using small-angle neutron scattering [16]. Note that the conical peaks are not visible in this scattering geometry as their $\mathbf{q}$ is along the beam. Further, increasing the field to $90 \mathrm{mT}$, a phase transition into the skyrmion state is observed [Fig. 1(d)]. For this magnetic history, the skyrmion state is initially a multidomain state, which transforms into a single domain skyrmion state at higher fields.

Next, we determine the $\mathbf{q}$ dependence of the scattering on the applied field and temperature by first integrating over the radial distribution of the scattered intensity and then fitting a series of Lorentzians to the respective data sets. These Lorentzians are parameterized by peak position, amplitude, and width. Plots of the scattered intensity and peak position (i.e., $|\mathbf{q}|)$ as a function of field and temperature are shown in Fig. 2. The phase diagrams on the left [Figs. 2(a) and 2(c)] show intensity and $|\mathbf{q}|$, respectively, for the scattered intensity integrated over the majority of the ring in Figs. 1(b)-1(d). They cover the well-known helical phase, as well as the skyrmion lattice phase (SkL) for higher fields, and are labeled 'Hel-I' and 'SkL.' The phase diagrams on the right [Figs. 2(b) and 2(d)] show intensity and $|\mathbf{q}|$ obtained for an integration over a $10^{\circ}$ region of interest around the horizontal, i.e., capturing only the two peaks marked by arrows in Fig. 1(c). These features occur at a field in between the helical Hel-I and skyrmion phase at lower temperatures where the phases are clearly separated. The phase diagrams highlight the behavior of the additional helical phase and are labeled 'Hel-II.'

The scattered intensity in the 'Hel-I + SkL' phase diagram in Fig. 2(a) clearly shows two distinct regions of high intensity (red color coded) in which the system is in the helical or, at high field, in the skyrmion phase. Above $T_{\mathrm{C}} \approx 273 \mathrm{~K}$, the scattering intensity vanishes. The observed value of $T_{\mathrm{C}}$ agrees with the observations in thin films [10], and is slightly lower than the bulk value of $278.7 \mathrm{~K}$. The q dependence of the Hel-I phase [Fig. 2(c)] shows a decrease in $\mathbf{q}$ with increasing field, while temperature only has a small effect on $\mathbf{q}$. In contrast, in the SkL phase, which is also visible in Fig. 2(c), $\mathbf{q}$ is affected more strongly by both temperature and field. The Hel-II phase diagrams in Figs. 2(b) and 2(d) show intensity and $\mathbf{q}$ for the additional helical phase. $|\mathbf{q}|$ shows a similar field dependence to that of the dominant helical phase but at a lower value.

Figure 3 shows the dependence of the magnitude of the magnetic modulation vector $\mathbf{q}$ for the different magnetic 


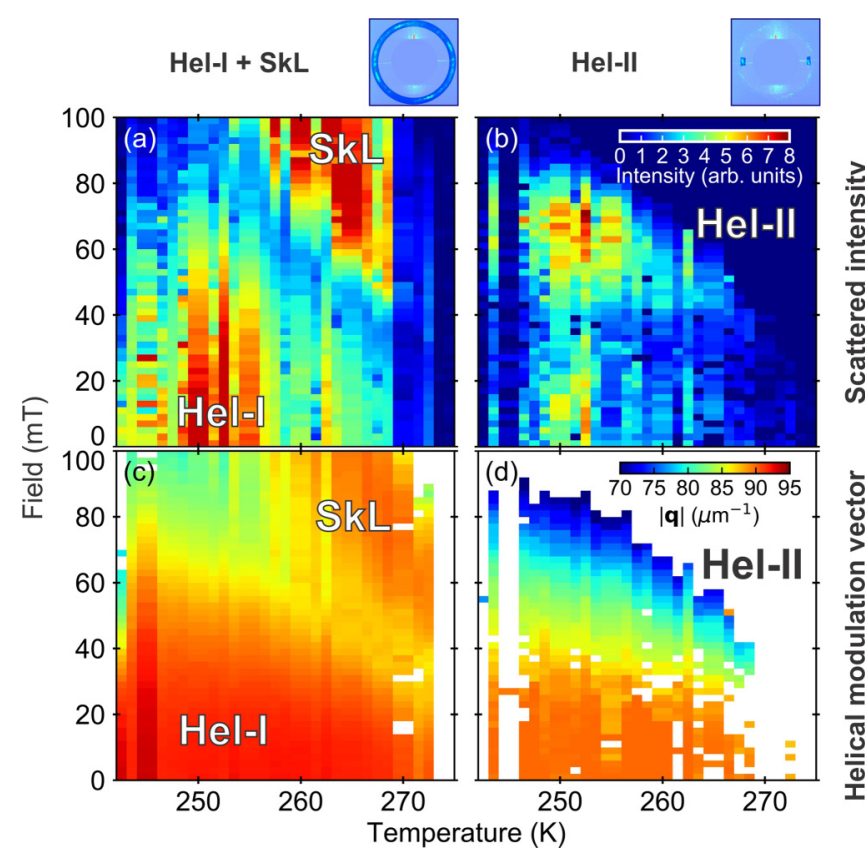

FIG. 2. Magnetic phase diagrams for (a),(b) the scattered intensity (top row) and (c),(d) the magnitude of the modulation vector q (bottom row) for the dominant helical phase (Hel-I and SkL, left column) and the additional helical phase (Hel-II, right column). In the left column, at higher fields, the skyrmion lattice phase (SkL) appears.

phases as a function of applied field for different temperatures (isotherms). The field values have been normalized to the upper critical field of the SkL phase pocket, $H_{c 2}$, which decreases with temperature (in line with the decrease of magnetization with temperature). After this renormalization, $\mathbf{q}$ of the helical phases is independent of temperature, as can be seen in Fig. 3, which shows coinciding isotherms.

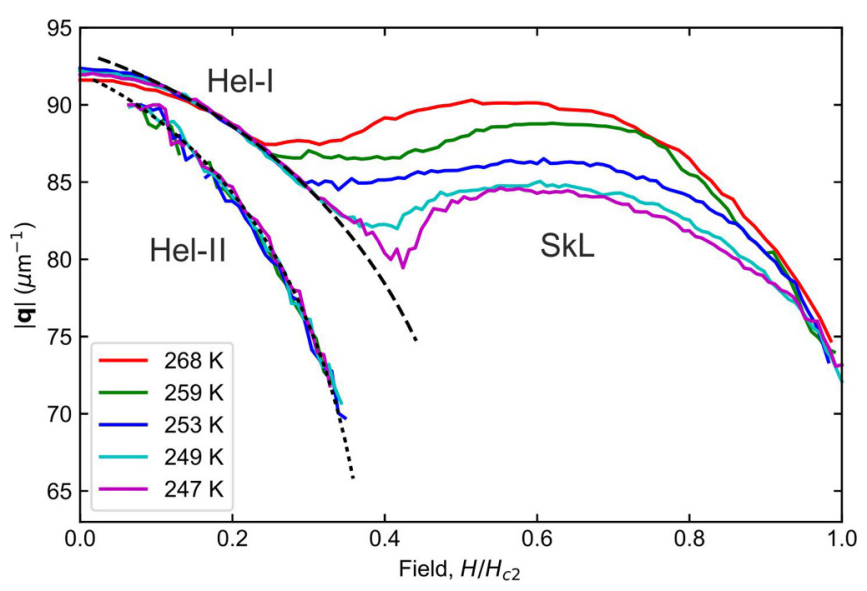

FIG. 3. Field dependence of $|\mathbf{q}|$ for helical and SkL phases for different temperatures. The field was normalized against the upper critical field $H_{c 2}$. Solid lines show the measured data (isotherms), while the dotted and dashed lines show the simulated $|\mathbf{q}|$ dependencies. Above: helical phase Hel-I and skyrmion phase SkL; below: additional helical phase Hel-II.
For both helical phases, q decreases with increasing applied field, i.e., the real-space helical structure expands, accompanied by a decrease in the scattered intensity. Overall, the $\mathbf{q}$ values of the additional Hel-II phase are smaller than those of the ordinary Hel-I phase. With a further increase in field the system transitions from the Hel-I phase to the skyrmion phase (for which $\mathbf{q}$ is both temperature and field dependent). The temperature, on the other hand, only has a minor effect on $\mathbf{q}$, as is different from the skyrmion phase.

For the two helical phases, the decrease of $\mathbf{q}$ with field means that the pitch of the proper screw magnetization structure increases. With the field applied perpendicular to $\mathbf{q}$, the width of regions within the structure with spins aligned with the field increases at the cost of those aligned opposite the field. This transition is accompanied by a net expansion of the lattice period. This behavior is reminiscent of the CSL observed, e.g., in $\mathrm{CrNb}_{3} \mathrm{~S}_{6}$ [14], showing a net expansion of the magnetization screw structure with field applied perpendicular to q. In this case the Zeeman energy favors the ferromagnetic alignment of spins with the field, however, the Dzyaloshinskii-Moriya interaction still prefers a chiral spin rotation. The combined effect gives a periodic distortion to the helical chiral structure which is well described through the effective one-dimensional chiral sine-Gordon model [23-28]. Following this method, the CSL period $L$ can be expressed as

$$
L(H)=8 K(\kappa) E(\kappa) /\left(\pi Q_{0}\right),
$$

where $K(\kappa)$ and $E(\kappa)$ are the complete elliptical integrals of the first and second kind, respectively, and $Q_{0}$ is the wave number of the helix.

The elliptic integrals are parameterized by the elliptic modulus $\kappa$ which takes the value $0<\kappa<1$. This value is found by energy minimization and relates to the applied magnetic field via $\kappa / K(\kappa)=\sqrt{H / H_{\mathrm{c}}}$. At the critical field, $H=H_{\mathrm{c}}$, the CSL transitions into the field-polarized state with no helical modulation and corresponds to $\kappa=1[E(1)=1]$. At zero field the helical state is not affected by periodic distortions, $\kappa=0$, and through the relations $K(0)=E(0)=\pi / 2$ the period from Eq. (1) simplifies to $L(0)=2 \pi / Q_{0}$.

Following a similar analysis by Togawa et al. [14], and by taking $\mathbf{q}(H) / \mathbf{q}(0)=L(0) / L(H)$, the dimensionless ratio of $\mathbf{q}$ for the CSL can be expressed as

$$
\mathbf{q}(H) / \mathbf{q}(0)=\pi^{2} /[4 K(\kappa) E(\kappa)] .
$$

Here, the change in $\mathbf{q}$ is temperature independent and can be entirely described by a change in $H$. The q varies from the helical pitch modulation at zero field and approaches 0 as $H$ approaches $H_{\mathrm{c}}$.

The dashed black lines in Fig. 3 show how the $\mathbf{q}$ dependence of both the Hel-I and Hel-II phases are accurately modeled by the expected $\mathbf{q}$ dependence from Eq. (2). In this figure, $\mathbf{q}(0)$ takes the value of 93.4 and $92.0 \mu \mathrm{m}^{-1}$ for Hel-I and Hel-II, respectively. Note that the CSL will give rise to higher harmonics of $\mathbf{q}$ (i.e., $2 \mathbf{q}, 3 \mathbf{q}, \ldots$ ) [19], however, with the detector distance optimized for high $q$ resolution, these additional peaks cannot be observed in our measurement.

Note that the field axis for Eq. (2) in Fig. 3 has been stretched by a factor of 0.55 and 0.40 for the two phases, respectively. The model in Eq. (2) uses a field normalized against a critical field at which the phase transforms into the 
field-polarized state (i.e., where all spins are aligned). The factors to adjust the field axis are likely to result from changes in this phase transition since we normalized against the upper critical field for the skyrmion phase pocket. Additionally, anisotropy in the thin slab of FeGe used in this investigation could provide an effective field in addition to the applied field also leading to a modification in the normalized field values. Note that the helical axis (q-vector direction) in $\mathrm{FeGe}$ also depends on temperature. In bulk crystals, it is along the [001] direction below $280 \mathrm{~K}$ and changes to the [111] direction at lower temperatures (from 211 to $245 \mathrm{~K}$ ) [1].

An anisotropy resulting from the shape of the thin-film slab could also explain why the effective field of the two helical phases is different. This would also be consistent with the additional Hel-II phase having a preferential ordering direction with respect to the geometry of the sample, while the Hel-I phase does not.

The observation that the temperature does not affect the helical phase (in the investigated temperature range near $T_{\mathrm{C}}$ ) is in contrast to measurements on $\mathrm{MnGe}[7,8,29,30]$ showing a significant temperature dependence of $\mathbf{q}$. Note that as the temperature is lowered significantly, the magnetic anisotropy change will affect the $\mathbf{q}$ of the helical phase.

\section{CONCLUSIONS}

In summary, we have studied the magnetic phase diagram of a 200-nm-thick FeGe film in out-of-plane fields using small-angle soft $\mathrm{x}$-ray elastic scattering. It was found that the modulation wave vector of the magnetic structures in the various phases shows a clear dependence on field strength and temperature. In the normal helical phase, the modulation period decreases with normalized field and is temperature independent. In contrast, in the skyrmion pocket at higher fields, $\mathbf{q}$ is a function of both field and temperature. The two additional scattering peaks give rise to an additional pocket in the phase diagram between the helical and skyrmion phases, showing some overlap with the helical phase. This feature has a stronger $\mathbf{q}$ dependence on the reduced field than the normal helical phase but otherwise follows the same trend and is temperature independent. The field dependence of the magnetic structure of both helical phases follows the model for a chiral soliton lattice. The deeper insight in the field dependence of chiral spin structures, in combination with magnetoresistance effects that are intimately connected to the number of kinks, could lead to novel nonvolatile memory devices in the future.

\section{ACKNOWLEDGMENTS}

The SAREXS experiments were carried out using the portable octupole magnet system on beamline I10 at the Diamond Light Source, U.K., under proposal SI-20437. S.L.Z. acknowledges a starting grant from ShanghaiTech University. Financial support through Engineering and Physical Sciences Research Council (EPSRC) Grant No. EP/N032128/1 is gratefully acknowledged.
[1] B. Lebech, J. Bernhard, and T. Freltoft, Magnetic structures of cubic FeGe studied by small-angle neutron scattering, J. Phys.: Condens. Matter 1, 6105 (1989).

[2] H. Wilhelm, M. Baenitz, M. Schmidt, U. K. Rößler, A. A. Leonov, and A. N. Bogdanov, Precursor Phenomena at the Magnetic Ordering of the Cubic Helimagnet FeGe, Phys. Rev. Lett. 107, 127203 (2011).

[3] J. C. Gallagher, K. Y. Meng, J. T. Brangham, H. L. Wang, B. D. Esser, D. W. McComb, and F. Y. Yang, Robust Zero-Field Skyrmion Formation in FeGe Epitaxial Thin Films, Phys. Rev. Lett. 118, 027201 (2017).

[4] Y. Tokunaga, X. Z. Yu, J. S. White, H. M. Rønnow, D. Morikawa, Y. Taguchi, and Y. Tokura, A new class of chiral materials hosting magnetic skyrmions beyond room temperature, Nat. Commun. 6, 7638 (2015).

[5] S. Mühlbauer, B. Binz, F. Jonietz, C. Pfleiderer, A. Rosch, A. Neubauer, R. Georgii, and P. Böni, Skyrmion lattice in a chiral magnet, Science 323, 915 (2009).

[6] A. Bauer, M. Garst, and C. Pfleiderer, Specific Heat of the Skyrmion Lattice Phase and Field-Induced Tricritical Point in MnSi, Phys. Rev. Lett. 110, 177207 (2013).

[7] N. Kanazawa, Y. Onose, T. Arima, D. Okuyama, K. Ohoyama, S. Wakimoto, K. Kakurai, S. Ishiwata, and Y. Tokura, Large Topological Hall Effect in a Short-Period Helimagnet MnGe, Phys. Rev. Lett. 106, 156603 (2011).

[8] O. L. Makarova, A. V. Tsvyashchenko, G. Andre, F. Porcher, L. N. Fomicheva, N. Rey, and I. Mirebeau, Neutron diffraction study of the chiral magnet MnGe, Phys. Rev. B 85, 205205 (2012).

[9] A. Bauer and C. Pfleiderer, Generic aspects of skyrmion lattices in chiral magnets, Springer Ser. Mater. Sci. 228, 1 (2016).

[10] X. Z. Yu, N. Kanazawa, Y. Onose, K. Kimoto, W. Z. Zhang, S. Ishiwata, Y. Matsui, and Y. Tokura, Near room-temperature formation of a skyrmion crystal in thin-films of the helimagnet FeGe, Nat. Mater. 10, 106 (2011).

[11] H. Wilhelm, M. Baenitz, M. Schmidt, C. Naylor, R. Lortz, U. K. Rößler, A. A. Leonov, and A. N. Bogdanov, Confinement of chiral magnetic modulations in the precursor region of $\mathrm{FeGe}$, J. Phys.: Condens. Matter 24, 294204 (2012).

[12] K. Shibata, J. Iwasaki, N. Kanazawa, S. Aizawa, T. Tanigaki, M. Shirai, T. Nakajima, M. Kubota, M. Kawasaki, H. S. Park, D. Shindo, N. Nagaosa, and Y. Tokura, Large anisotropic deformation of skyrmions in strained crystal, Nat. Nanotechnol. 10, 589 (2015).

[13] S. X. Huang and C. L. Chien, Extended Skyrmion Phase in Epitaxial FeGe Thin Films, Phys. Rev. Lett. 108, 267201 (2012).

[14] Y. Togawa, T. Koyama, K. Takayanagi, S. Mori, Y. Kousaka, J. Akimitsu, S. Nishihara, K. Inoue, A. S. Ovchinnikov, and J. Kishine, Chiral Magnetic Soliton Lattice on a Chiral Helimagnet, Phys. Rev. Lett. 108, 107202 (2012).

[15] Y. Togawa, Y. Kousaka, K. Inoue, and J. I. Kishine, Symmetry, structure, and dynamics of monoaxial chiral magnets, J. Phys. Soc. Jpn. 85, 112001 (2016). 
[16] N. Kanazawa, J. S. White, H. M. Rønnow, C. D. Dewhurst, Y. Fujishiro, A. Tsukazaki, Y. Kozuka, M. Kawasaki, M. Ichikawa, F. Kagawa, and Y. Tokura, Direct observation of anisotropic magnetic field response of the spin helix in FeGe thin films, Phys. Rev. B 94, 184432 (2016).

[17] Y. Yamasaki, D. Morikawa, T. Honda, H. Nakao, Y. Murakami, N. Kanazawa, M. Kawasaki, T. Arima, and Y. Tokura, Dynamical process of skyrmion-helical magnetic transformation of the chiral-lattice magnet FeGe probed by small-angle resonant soft X-ray scattering, Phys. Rev. B 92, 220421(R) (2015).

[18] Y. Okamura, Y. Yamasaki, D. Morikawa, T. Honda, V. Ukleev, H. Nakao, Y. Murakami, K. Shibata, F. Kagawa, S. Seki, T. Arima, and Y. Tokura, Directional electric-field induced transformation from skyrmion lattice to distinct helices in multiferroic $\mathrm{Cu}_{2} \mathrm{OSeO}_{3}$, Phys. Rev. B 95, 184411(R) (2017).

[19] Y. Okamura, Y. Yamasaki, D. Morikawa, T. Honda, V. Ukleev, H. Nakao, Y. Murakami, K. Shibata, F. Kagawa, S. Seki, T. Arima, and Y. Tokura, Emergence and magnetic-field variation of chiral-soliton lattice and skyrmion lattice in the strained helimagnet $\mathrm{Cu}_{2} \mathrm{OSeO}_{3}$, Phys. Rev. B 96, 174417 (2017).

[20] V. Ukleev, Y. Yamasaki, D. Morikawa, K. Karube, K. Shibata, Y. Tokunaga, Y. Okamura, K. Amemiya, M. Valvidares, H. Nakao, Y. Taguchi, Y. Tokura, and T. Arima, Element-specific soft $\mathrm{x}$-ray spectroscopy, scattering, and imaging studies of the skyrmion-hosting compound $\mathrm{Co}_{8} \mathrm{Zn}_{8} \mathrm{Mn}_{4}$, Phys. Rev. B 99, 144408 (2019).

[21] H. Du, X. Zhao, F. N. Rybakov, A. B. Borisov, S. Wang, J. Tang, C. Jin, C. Wang, W. Wei, N. S. Kiselev, Y. Zhang, R. Che, S. Blügel, and M. Tian, Interaction of Individual Skyrmions in a Nanostructured Cubic Chiral Magnet, Phys. Rev. Lett. 120, 197203 (2018).
[22] H. Du, R. Che, L. Kong, X. Zhao, C. Jin, C. Wang, J. Yang, W. Ning, R. Li, C. Jin, X. Chen, J. Zang, Y. Zhang, and M. Tian, Edge-mediated skyrmion chain and its collective dynamics in a confined geometry, Nat. Commun. 6, 8504 (2015).

[23] J.-I. Kishine, K. Inoue, and Y. Yoshida, Synthesis, structure and magnetic properties of chiral molecule-based magnets, Prog. Theor. Phys. Supp. 159, 82 (2007).

[24] A. B. Borisov, J.-I. Kishine, I. G. Bostrem, and A. S. Ovchinnikov, Magnetic soliton transport over topological spin texture in chiral helimagnet with strong easy-plane anisotropy, Phys. Rev. B 79, 134436 (2009).

[25] I. G. Bostrem, J.-I. Kishine, and A. S. Ovchinnikov, Theory of spin current in chiral helimagnets, Phys. Rev. B 78, 064425 (2008).

[26] J.-I. Kishine and A. S. Ovchinnikov, Theory of spin resonance in a chiral helimagnet, Phys. Rev. B 79, 220405(R) (2009).

[27] J.-I. Kishine, A. S. Ovchinnikov, and I. V. Proskurin, Sliding conductivity of a magnetic kink crystal in a chiral helimagnet, Phys. Rev. B 82, 064407 (2010).

[28] J.-I. Kishine, I. V. Proskurin, and A. S. Ovchinnikov, Tuning Magnetotransport Through a Magnetic Kink Crystal in a Chiral Helimagnet, Phys. Rev. Lett. 107, 017205 (2011).

[29] T. Tanigaki, K. Shibata, N. Kanazawa, X. Yu, Y. Onose, H. S. Park, D. Shindo, and Y. Tokura, Real-space observation of short-period cubic lattice of skyrmions in MnGe, Nano Lett. 15, 5438 (2015).

[30] N. Kanazawa, J.-H. Kim, D. S. Inosov, J. S. White, N. Egetenmeyer, J. L. Gavilano, S. Ishiwata, Y. Onose, T. Arima, B. Keimer, and Y. Tokura, Possible skyrmion-lattice ground state in the B20 chiral-lattice magnet MnGe as seen via smallangle neutron scattering, Phys. Rev. B 86, 134425 (2012). 